\title{
PET/CT for staging lung cancer: costly or cost-saving?
}

\author{
Andreas K. Buck • Ken Herrmann • Jonas Schreyögg
}

Received: 10 March 2011 / Accepted: 14 March 2011 / Published online: 26 March 2011

(C) Springer-Verlag 2011

Integrated functional and anatomical imaging using positron emission tomography (PET)/CT hybrid scanners has changed the diagnostic algorithm in non-small cell lung cancer (NSCLC). The accuracy for detection of lymph node and distant organ metastases is superior to separately performed PET or CT scanning and other invasive or noninvasive diagnostic tests [1]. There is also evidence that PET/CT is particularly effective if curative surgery is cancelled when $\mathrm{PET} / \mathrm{CT}$ indicates advanced disease whereas conventional staging procedures suggest curable disease. Diagnostic effectiveness of PET/CT for staging NSCLC and differential diagnosis of solitary pulmonary nodules has been recently approved by many health care systems worldwide. Additional indications for cancer imaging which are recognized in the USA and several European countries include staging and restaging of oesophageal, colorectal and breast cancer, malignant lymphoma, melanoma and head and neck cancer [2]. Regarding these indications, a diagnostic effectiveness and superiority to conventional cancer imaging modalities has been demonstrated. However, if PET/CT is

\footnotetext{
A. K. Buck ( $\square)$

Dept. of Nuclear Medicine, Nuklearmedizinische Klinik und Poliklinik, Universitätsklinikum Würzburg,

Oberdürrbacherstr. 6,

97080 Würzburg, Germany

e-mail: andreas.buck@tum.de

\section{K. Herrmann}

Dept. of Nuclear Medicine, Technische Universität München, Munich, Germany

J. Schreyögg

Institute of Health Economics and Health Care Management, Universität Hamburg,

Fakultät für Wirtschafts- und Sozialwissenschaften, Lehrstuhl für Management im Gesundheitswesen,

Hamburg, Germany
}

also cost-effective when integrated into routine diagnostic algorithms has never been demonstrated. In lung cancer, noninvasive imaging modalities contribute to only a small proportion of total health care costs $(\sim 5-6 \%)$ [3]. However, given the limited resources of health care systems, evidence for economic efficiency of novel diagnostic tools or therapeutics will become more relevant in the near future.

In this issue of the European Journal of Nuclear Medicine and Molecular Imaging, Søgaard et al. report on the costeffectiveness of PET/CT for staging NSCLC [4]. In a randomized clinical trial, PET/CT was performed in addition to the conventional staging procedures in 98 patients. In the control group consisting of 91 patients, conventional staging was performed without PET/CT. They have shown that implementation of PET/CT into the diagnostic algorithm of staging NSCLC is cost-effective. The incremental costeffectiveness ratio (ICER) was calculated to be $19,314 €$, meaning that $\mathrm{PET} / \mathrm{CT}$ is cost-effective if the provider's willingness to pay is $50,000 €$ per avoided 'futile' thoracotomy. The incremental cost for adding PET/CT to the diagnostic workup was estimated to be as high as $3,927 €$. A more detailed analysis of cancer-related and comorbidity-related costs indicated that in the PET/CT group unexpectedly high comorbidity-related costs were spent in four patients which were not adequately randomized between both groups. When these comorbidity-related costs are excluded from the analysis, PET/CT appears to be dominant over routine diagnostic workup, resulting in cost savings of $899 €$ per patient and savings of $4,495 €$ per avoided thoracotomy.

In the same study collective, the authors recently assessed the effect of combined PET/CT imaging on preoperative staging of NSCLC [5]. The number of patients identified as having advanced disease is twice as high as compared to the conventional group and, subsequently, a significantly higher number of non-curative, thus, unnecessary surgical 
interventions were performed in the conventional staging group. A gain in diagnostic accuracy when PET/CT is used for staging NSCLC instead of CT scanning has already been demonstrated by Lardinois et al. [1], Halpern et al. [6] and Shim et al. [7] and was also observed in the present study. Interestingly, PET/CT did not impact survival which, after clinical follow-up for 1 year, was similar in both groups. Similar to previous trials reporting on the diagnostic accuracy of dedicated PET (without coregistration of CT) [8], about five PET/CT scans have to be performed to avoid one futile thoracotomy.

This manuscript is one of the very first assessing costeffectiveness of PET/CT for staging NSCLC, providing further evidence that PET/CT is not only clinically effective but also economically efficient. A major strength of the study is that cost-effectiveness was analysed alongside a randomized controlled clinical trial, a study design which is scarcely found in clinical studies evaluating noninvasive imaging modalities. Moreover, a high number of 192 patients were prospectively recruited. A full health care sector perspective was used and real costs were calculated rather than estimation of prices, including all expenses related to cancer and comorbidities, making their findings even more suitable to estimate the economic value of PET/CT.

Cost-effectiveness of PET and PET/CT for staging lung cancer is based on the potential of metabolic imaging to detect metastases at anatomical sites which appear normal or benign at morphology based imaging modalities such as CT or MRI. Prevention of unnecessary surgical interventions has been reported to occur in up to $14 \%$ of patients [9]. Recently, a randomized study has shown that the number of futile thoracotomies was significantly reduced in $19 / 82$ patients compared to $29 / 96$ patients, based on the addition of fluorodeoxyglucose (FDG) PET to the diagnostic algorithm [8]. The present study by Søgaard et al. showed similar results with 21/98 futile thoracotomies in the PET/CT group compared to 38/91 surgical interventions in the control group which were interpreted as futile. The relative risk of futile thoracotomy was as low as 0.21 in the PET/CT group compared to 0.42 in the conventional staging group.

Before the onset of hybrid PET/CT systems, the costeffectiveness of dedicated PET scanners had already been demonstrated by two independent studies [10, 11]. Both groups could demonstrate that PET is cost-effective if mediastinal lymph node metastases can be detected in a patient with a normal chest CT, without evidence of suspicious nodes. This is the case in up to $20 \%$ of patients with an initial diagnosis of lung cancer. Accordingly, a significantly lower number of patients undergo resective surgery with curative intent. Conversely, patients with mediastinal nodes suspicious at CT but negative at PET or $\mathrm{PET} / \mathrm{CT}$ do not have to undergo additional mediastinoscopy. Costs for mediastinoscopy can therefore be avoided. In the case of a resectable primary tumour, these patients can directly undergo resective surgery performed with curative intent. In cases of FDG-positive nodes, however, mediastinoscopy is recommended to rule out false-positive findings [12]. Additional costs may evolve from PET/CT and the contribution to cost savings is not significant in cases of FDG-positive mediastinal nodes. In the study by Søgaard et al., all patients underwent mediastinoscopy which is not mandatory. Therefore, additional cost savings could not be implemented in the PET/CT group. Overall, the use of PET and PET/CT for staging NSCLC has been shown to be cost-effective in several trials. Recently, the diagnostic value and costeffectiveness have also been approved by the German health care system (Federal Joint Committee, G-BA).

Several limitations have to be considered when transferring these findings to the general clinical situation. First of all, this is not a dedicated cost-effectiveness study. The primary aim was to evaluate the clinical benefit of PET/CT in terms of reduction of the number of futile thoracotomies, whereas analysis of cost-effectiveness seems to represent a secondary aim. Another prominent limitation applies to the fact that cost-effectiveness analysis has been performed which does not reflect the societal perspective of health costs. Although an unexpectedly high amount of comorbidity-related costs wase found in the PET/CT group, it does not seem appropriate in a randomized trial to turn a surplus of $3,927 €$ into savings of $899 €$ by ignoring the comorbidity-related costs. Therefore, the relevant findings are the reported incremental costs of $3,927 €$ in the PET/CT group.

In the present study, follow-up was restricted to 12 months which seems rather short for assessing outcome of NSCLC. Moreover, criteria for determining when a thoracotomy is considered futile are still a matter of debate. This adds significant bias to the economic calculations performed in this study. Even in a palliative situation, resective surgery may be beneficial to the patient, e.g. for restoration of local complications. Furthermore, FDG-avid lesions were not validated in all patients; hence, falsepositive findings with a potential impact on patient outcome cannot be excluded. Given the paucity of available data, the work is important and clearly illustrates the need for future studies evaluating the cost-effectiveness of PET/CT, considering also emerging diagnostic modalities such as endobronchial ultrasound (EBUS) for mediastinal staging of NSCLC. Suitable designs for future clinical trials assessing cost-effectiveness or analysing cost-utility and cost-benefit of PET/CT for cancer imaging have been recently suggested [2].

Besides the publication of Søgaard et al., there is only one research paper reporting cost-effectiveness of PET/CT for staging NSCLC. In our own study comprising 172 patients with an initial diagnosis of NSCLC, the ICER was 
in a similar range as reported by Søgaard et al. (3,810€ per patient correctly staged as resectable vs non-resectable) [13]. In addition, a cost-utility analysis was performed. The ICER per quality-adjusted life year gained was $63,617 €$ which is only slightly above an accepted threshold of $50,000 €$ for reimbursement acceptance which is frequently used as a reference by health technology assessment agencies. Thus, the results of Søgaard et al. and the findings of our cost-effectiveness and cost-utility analyses suggest that in industrialized countries PET/CT for staging of NSCLC should be reimbursed by public health care systems.

Nevertheless, PET and more recently PET/CT still struggle to get funded by national health care systems. From a payer's perspective, concerns regarding a widespread utilization of PET/CT for cancer imaging are reasonable. Therefore, future clinical trials specifically designed to assess the cost-effectiveness of PET/CT at least in the more frequent tumour entities are highly warranted.

\section{References}

1. Lardinois D, Weder W, Hany TF, Kamel EM, Korom S, Seifert B, et al. Staging of non-small-cell lung cancer with integrated positron-emission tomography and computed tomography. N Engl J Med 2003;348(25):2500-7.

2. Buck AK, Herrmann K, Stargardt T, Dechow T, Krause BJ, Schreyögg J. Economic evaluation of PET and PET/CT in oncology: evidence and methodologic approaches. J Nucl Med 2010;51(3):401-12.

3. Dinan MA, Curtis LH, Hammill BG, Patz EF, Abernethy AP, Shea AM, et al. Changes in the use and costs of diagnostic imaging among Medicare beneficiaries with cancer, 1999-2006. JAMA 2010;303(16):1625-31.
4. Søgaard R, Fischer BM, Mortensen J, Højgaard L, Lassen U. Preoperative staging of lung cancer with PET/CT: costeffectiveness evaluation alongside a randomized controlled trial. Eur J Nucl Med Mol Imaging 2011. doi:10.1007/s00259-0101703-y.

5. Fischer B, Lassen U, Mortensen J, Larsen S, Loft A, Bertelsen A, et al. Preoperative staging of lung cancer with combined PET-CT. N Engl J Med 2009;361(1):32-9.

6. Halpern BS, Schiepers C, Weber WA, Crawford TL, Fueger BH, Phelps ME, et al. Presurgical staging of non-small cell lung cancer: positron emission tomography, integrated positron emission tomography/CT, and software image fusion. Chest 2005;128 (4):2289-97.

7. Shim SS, Lee KS, Kim BT, Chung MJ, Lee EJ, Han J, et al. Nonsmall cell lung cancer: prospective comparison of integrated FDG $\mathrm{PET} / \mathrm{CT}$ and $\mathrm{CT}$ alone for preoperative staging. Radiology 2005;236(3):1011-9.

8. van Tinteren H, Hoekstra OS, Smit EF, van den Bergh JH, Schreurs AJ, Stallaert RA, et al. Effectiveness of positron emission tomography in the preoperative assessment of patients with suspected non-small-cell lung cancer: the PLUS multicentre randomised trial. Lancet 2002;359(9315):1388-93.

9. Pieterman RM, van Putten JW, Meuzelaar JJ, Mooyaart EL, Vaalburg W, Koëter GH, et al. Preoperative staging of non-smallcell lung cancer with positron-emission tomography. $\mathrm{N}$ Engl $\mathrm{J}$ Med 2000;343(4):254-61.

10. Dietlein M, Weber K, Gandjour A, Moka D, Theissen P, Lauterbach KW, et al. Cost-effectiveness of FDG-PET for the management of potentially operable non-small cell lung cancer: priority for a PET-based strategy after nodal-negative CT results. Eur J Nucl Med 2000;27(11):1598-609.

11. Farjah F, Flum DR, Ramsey SD, Heagerty PJ, Symons RG, Wood DE. Multi-modality mediastinal staging for lung cancer among medicare beneficiaries. J Thorac Oncol 2009;4(3):355-63.

12. Detterbeck FC, Jantz MA, Wallace M, Vansteenkiste J, Silvestri GA, American College of Chest Physicians. Invasive mediastinal staging of lung cancer: ACCP evidence-based clinical practice guidelines (2nd edition). Chest 2007;132(3 Suppl):202S-20.

13. Schreyögg J, Weller J, Stargardt T, Herrmann K, Bluemel C, Dechow T, et al. Cost-effectiveness of hybrid PET/CT for staging of non-small cell lung cancer. J Nucl Med 2010;51(11):1668-75. 